\title{
Improving the Performance of Wireless Ad Hoc Networks Through MAC Layer Design
}

\author{
Mariam Kaynia, Student Member, IEEE, Nihar Jindal, Member, IEEE, \\ and Geir E. Øien, Senior Member, IEEE
}

\begin{abstract}
In this paper, the performance of the ALOHA and CSMA MAC protocols are analyzed in spatially distributed wireless networks. The main system objective is correct reception of packets, and thus the analysis is performed in terms of outage probability. In our network model, packets belonging to specific transmitters arrive randomly in space and time according to a 3-D Poisson point process, and are then transmitted to their intended destinations using a fully-distributed MAC protocol. A packet transmission is considered successful if the received SINR is above a predefined threshold for the duration of the packet. Accurate bounds on the outage probabilities are derived as a function of the transmitter density, the number of backoffs and retransmissions, and in the case of CSMA, also the sensing threshold. The analytical expressions are validated with simulation results. For continuous-time transmissions, CSMA with receiver sensing (which involves adding a feedback channel to the conventional CSMA protocol) is shown to yield the best performance. Moreover, the sensing threshold of CSMA is optimized. It is shown that introducing sensing for lower densities (i.e., in sparse networks) is not beneficial, while for higher densities (i.e., in dense networks), using an optimized sensing threshold provides significant gain.
\end{abstract}

Index Terms-Ad hoc networks, Poisson point process, MAC protocols, outage probability.

\section{INTRODUCTION}

$\mathbf{I}$ $\mathrm{N}$ the design of wireless ad hoc networks, various techniques are applied to efficiently allocate the scarce resources available for the communication links. Using an appropriate medium access control (MAC) protocol is one such technique. Taking into account the system's quality of service (QoS) requirements, a MAC protocol for ad hoc networks shares the medium and the available resources in a distributed manner, and allows for efficient interference management.

In this paper, we consider a spatial network model in which nodes are randomly distributed in space, and we address the problem of interference through MAC layer design. The ALOHA and CSMA MAC protocols are employed for communication, and the success rate of packet transmissions is investigated. In particular, we ask the following questions: (a) Given a fixed signal-to-interference-plus-noise ratio (SINR) threshold for each transmitter (TX) receiver (RX) link in the

Manuscript received March 3, 2010; revised June 29, 2010 and October 7, 2010; accepted October 8, 2010. The associate editor coordinating the review of this paper and approving it for publication was O. Dabeer.

M. Kaynia and G. E. Øien are with the Dept. of Electronics and Telecommunications, Norwegian University of Science and Technology, Trondheim, Norway (e-mail: \{kaynia, oien\}@iet.ntnu.no).

N. Jindal is with the Dept. of Electrical and Computer Engineering, University of Minnesota, MN, USA (e-mail: nihar@umn.edu).

Digital Object Identifier 10.1109/TWC.2010.110310.100316 network, what is the probability of successful transmission for ALOHA and CSMA, (b) can the performance of CSMA be improved by introducing feedback between the TX and RX and allowing the RX to make the backoff decision, and (c) does CSMA have an optimal sensing threshold which minimizes the outage probability (OP) for received packets?

We consider a network in which packets are located randomly in space and time according to a 3-D Poisson point process (PPP), consisting of a 2-D PPP of TX locations in space and a 1-D PPP of packet arrivals in time. The packets, which are assumed to be of constant length, are forwarded by each TX over a nonfading channel to a RX a fixed distance away. In order to derive precise results, we focus exclusively on single-hop communication, as in [1], [2], [3]. All multiuser interference is treated as noise, and our model uses the SINR to evaluate the performance (in terms of OP) of the communication system. The only source of randomness in the model is in the location of nodes and concurrent transmissions, which allows us to focus on the relationships between transmission density, OP, sensing threshold, and the choice of MAC protocol.

\section{A. Related Work}

There has been a notable amount of research done on the performance of ALOHA in ad hoc networks. A number of researchers have analyzed slotted ALOHA using a Poisson model for TX locations, considering transmission capacity and success probability of the network [2], [4], [5]. Ferrari and Tonguz [6] have analyzed the transport capacity of slotted ALOHA and CSMA, showing that for low transmission densities the performance of slotted ALOHA is almost twice that of CSMA. Also, it is established that CSMA is advantageous only at high transmission densities. Other related works have considered the performance of ALOHA and CSMA in terms of throughput and bit error rate [6], [7], [8]. Some of these also assert CSMA's superiority over ALOHA, which is naturally followed by tradeoffs in other domains such as transmission rate and delay [8] [9]. The seminal work of Gupta and Kumar [7] considers the transport capacity of a Poisson distributed ad hoc network, which resembles a slotted version of our model. However, their analysis focuses on a deterministic SINR model, and employs a deterministic channel access scheme, thereby precluding the occurrence of outages. Weber et al. [5] revise this model by considering a stochastic SINRbased model, within which they find tight lower and upper bounds to the OP of slotted ALOHA as a function of the 
node density. We consider the model used in [5], and extend it to also cover unslotted systems.

Despite all the research done on MAC protocols thus far, only a limited number of works have considered an interference channel that is both stochastic and continuous in time [2], [4], [5], [10]. Perhaps the closest work is that of Hasan and Andrews [4], where the success probability of slotted ALOHA is analyzed within such a stochastic ad hoc wireless network model. Success probability is defined as the probability that a transmission is received successfully at the $\mathrm{RX}$. This is equal to $1-\mathrm{OP}$. In [4], a scheduling mechanism is assumed that creates an interferer-free guard zone, which is in effect a theoretical circle around the RX, within which no interfering TXs are allowed. By means of geometrical analysis, the density of successful transmissions is maximized under an outage constraint. We adopt the concept of guard zones in our analysis, with the difference that instead of incorporating into the protocol a guard zone within which no TX are permitted, we consider actual MAC protocols that employ virtual guard zones in order to make the backoff decision and evaluate the OP. Other analytical models may also be used for performance evaluation [11], [12]. In [11], the throughput and fairness of CSMA/CA is evaluated based on a Markovian analysis. In [12], an analytical framework is introduced to evaluate the per-flow throughput of CSMA in a multi-hop environment. However, in both of these works, a single communication link is considered. Thus, the complexity of various links' performance and decisions being inter-dependent, as in interference channels, is ignored. This is considered in our model.

A good choice of the sensing threshold of CSMA, $\beta_{\text {sens }}$, is of great importance for its performance. In [13], the throughput of the CSMA protocol is evaluated in a multi-hop ad hoc network. It is shown that the optimal algorithm is to decrease the sensing range as long as the network remains sufficiently connected. In [14], it is claimed that in order to maximize the spatial reuse of the network, one must set $\beta_{\text {sens }} \approx \rho\left(1+\beta_{\text {req }}^{1 / \alpha}\right)^{\alpha}$, where $\rho$ represents the transmitted signal strength, $\alpha$ is the path loss exponent, and $\beta_{r e q}$ is the minimum required SINR threshold for correct packet reception. In [15], it is shown that the optimum sensing threshold of CSMA depends on the system design parameters, such as the distance between a TX and its RX. The authors conclude that to minimize the OP, senders must keep the product of their transmit power and carrier sensing threshold equal to a fixed constant. However, this algorithm is not distributed, and is dependent on estimation of signal powers. Loosening these limitations, a new sensing threshold adaptation algorithm is proposed in [16], where each node chooses the $\beta_{\text {sens }}$ that maximizes the number of successful transmissions in its neighborhood. The drawback of this scheme is that it relies on the collection of information from the environment over a period of time, which entails high complexity and is not able to handle fast variations of the interference.

The rest of this paper is organized as follows. In Section II, we provide a detailed description of the system model. In Sections III and IV, the OP of ALOHA and CSMA are derived. In Section V, we allow the sensing threshold of CSMA to vary, and find the optimal sensing threshold that minimizes the OP. Section VI presents the numerical results, while Section VII concludes the paper.

\section{SySTEM Model}

As a starting point, consider a mobile wireless network in which TXs are randomly placed on an infinite 2-D plane according to a homogeneous PPP with spatial density $\lambda^{s}$ [nodes $/ \mathrm{m}^{2}$ ] and moving independently of each other. At each TX, a series of packets, each with a fixed duration $T$, arrives according to an independent homogeneous 1-D PPP in time with intensity $\lambda^{t}$ [packets/sec/node]. These packets are then sent with a constant power $\rho$ to the intended RX, which is assumed to lie a fixed distance $R$ away. Futhermore, each packet is given $M$ backoffs and $N$ retransmission attempts. In this network model, the spatial PPP is first fixed and each TX generates its own traffic of packets. This means that at each time instant, the average number of new packets that are active per unit area is $\lambda=\lambda^{s} \lambda^{t} T$ [packets $/ \mathrm{m}^{2}$ ]. Due to the ability to backoff from transmissions (in CSMA) and retransmit in the case of erroneous packet reception, we have an increase of $\lambda \Delta(M, N)$ to the spatial density. Hence, the density of packets attempting to access the channel at each time instant is $\lambda(1+\Delta(M, N))$. If no backoffs or retransmissions are allowed, i.e., $(M, N)=(1,0)$, we have that $\Delta(M, N)=0$ for all the protocols. Note that the number of backoffs $M$ is always strictly greater than 0 .

Since this network model entails two independent Poisson distributions, in order to derive the OP, we would have to average over both the spatial and temporal statistics. Due to the complicated analysis this would entail, instead we consider our wireless network from an alternative point of view; Consider a single queue of packet arrivals with density $\lambda^{s} \lambda^{t} A$, where $A$ is the area of the communication region. Upon the arrival of each packet, it is assigned to a TX node, which is then randomly placed on a 2-D plane (uniformly distributed in area $A$ ), as illustrated in Fig. 1. The transmission to the intended $\mathrm{RX}$ is then initiated according to the specified MAC protocol. When the packet has been served (successfully or not), the corresponding TX-RX pair disappears from the plane. When the maximum number of backoffs and retransmissions is not reached, the packet is placed back in the packet arrival queue, with a new transmission time. The retransmitted packet will be located in a new position, which is justified by our assumption on high mobility. Considering the increase in the density of packets attempting to access the channel, we have that $\lambda=$ $\lambda^{s} \lambda^{t} T(1+\Delta(M, N))$.

Note that the temporal PPP of packet arrivals at each node is independent of the PPP of TX locations in space. Due to the high mobilility presumed in our network, different sets of packets are active between times $t_{0}$ and $t_{0}+T$. Since the waiting time from one transmission attempt to the next is set to be greater than $T$, we have that there are no spatial and temporal correlations between retransmission attempts. This is in accordance with the results of [17]. As a result of this independence, and the basic properties of PPPs, we have that the number of nodes in any random selection of an area at any random point in time still follows a PPP [18]. Hence, we have that our space-time model entails a 3-D PPP with density $\lambda^{s} \lambda^{t}(1+\Delta(M, N))$ [packets $/ \mathrm{m}^{2} / \mathrm{sec}$ ]. Considering the 


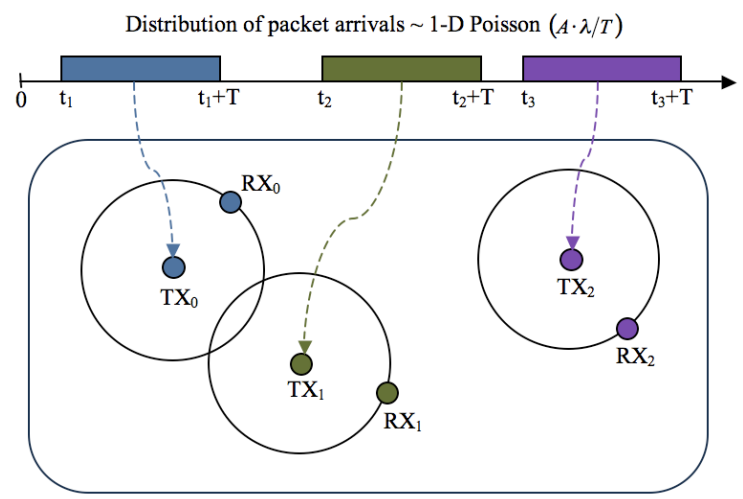

Fig. 1. Each new packet arrival is assigned to a TX-RX pair, which is then located randomly on a $2-\mathrm{D}$ plane.

entire plane at a fixed point in time, we observe the behaviour of the space-time model's spatial 2-D PPP with density $\lambda=$ $\lambda^{s} \lambda^{t} T(1+\Delta(M, N))$. Equivalently, the total expected number of packets in area $A$ during a time interval $T$ is $\lambda^{s} \lambda^{t} A T(1+$ $\Delta(M, N))$. Thus, we see that our alternative 3-D space-time model is a good representation of the ad hoc network initially described, as it entails a Poisson distribution of nodes in space and of packet arrivals in time, with the same density of packets accessing the channel as the initial model. Hence, we adopt this model for our analysis, as it allows us to only consider a single random process describing both the temporal and spatial variations of the system.

Note the following main attributes of our traffic model that are of significance in our derivations:

- Our network is highly mobile, meaning that different and independent sets of nodes are observed on the plane from one slot (of length $T$ ) to the next.

- Upon retransmission of a packet, it is treated as a new packet arrival and placed in a new location, resulting in no spatial correlation between transmission attempts.

- The waiting time between retransmission attempts is set to be $t_{\text {wait }}>T$, which because of the high mobility assumption, results in no temporal correlation between retransmission attempts.

For the channel model, we consider only path loss attenuation effects (with path loss exponent $\alpha>2$ ), ignoring both short term and long term fading. The channel is assumed to be constant for the duration of a transmission. Each RX sees interference from all active TXs on the plane, and these interference powers are added to the channel noise, $\eta$, to result in a certain SINR at each RX. If this received SINR falls below the required SINR threshold, $\beta_{\text {req }}$, at any time during the packet transmission, the packet is received erroneously and must be retransmitted, with probability

$$
P_{r t}=\operatorname{Pr}\left(\min _{0 \leq t \leq T} \frac{\rho R^{-\alpha}}{\eta+\sum_{i(t)} \rho r_{i}^{-\alpha}} \leq \beta_{r e q}\right),
$$

where $r_{i}$ is the distance between the node under observation and the $i$-th interfering TX, and the summation is over all active interferers on the plane at time $t$.

The MAC protocols ALOHA and CSMA are applied for communication between nodes. In the case of unslotted ALOHA, each transmission starts as soon as the packet arrives, regardless of the channel condition. Slotted ALOHA improves the performance by removing partial outages, but this requires synchronization. If the packet is received erroneously, it is retransmitted. Each packet has a maximum of $N$ retransmission attempts in order to be received correctly. In the CSMA protocol, the channel is sensed at the beginning of each packet. If the measured SINR is above $\beta_{\text {sens }}$, the packet transmission is initiated; otherwise, it is backed off. Each packet is given a maximum of $M$ backoffs, before it is dropped. Since evaluating the backoff scheme is outside the scope of this work, we simply assume that the backoff times are random, uncorrelated, and exponentially distributed (this also maintains the Poisson distribution of packets). Once the transmission is initiated, but the packet is received erroneously after $N$ retransmissions, it is counted to be in outage. Finally, all communication between the TX and its RX is assumed to occur over an orthogonal control channel, and the delay introduced by the feedback is assumed to be insignificant compared to the packet length.

In the general sense, the OP of ALOHA and CSMA is defined mathematically as

$$
\begin{aligned}
& P_{\text {out }}(\mathrm{ALOHA})= \\
& \quad \operatorname{Pr}\left[\mathrm{SINR}<\beta_{\text {req }} \text { at some } t \in[0, T) N+1 \text { times }\right] \\
& P_{\text {out }}(\mathrm{CSMA})=\operatorname{Pr}\left[\mathrm{SINR}<\beta_{\text {sens }} \text { at } t=0 M \text { times } \cup\right. \\
& \left.\quad \mathrm{SINR}<\beta_{\text {req }} \text { at some } t \in(0, T) N+1 \text { times }\right] .
\end{aligned}
$$

The throughput $S$ of this network is given as: $S=\lambda(1+$ $\Delta(M, N)) b\left(1-P_{\text {out }}\right)\left[\mathrm{bits} / \mathrm{s} / \mathrm{Hz} / \mathrm{m}^{2}\right]$, where $b$ is the average rate that a successful packet achieves, with units $[\mathrm{bits} / \mathrm{s} / \mathrm{Hz}$ per packet]. Since the OP is the only unknown term in the throughput expression, we will solely focus on this metric in the remainder of this paper.

\section{A. Justification of Assumptions}

For ad hoc networks with single-hop communication links and substantial mobility or indiscriminate node placement, such as a dense sensor network, an assumption of Poisson distributed nodes in space is reasonable and commonly used [2], [3], [4]. However, in many networks, such as clustered or multi-hop networks, this assumption might not be valid anymore, as transmissions are often correlated with each other in both time and space. Assuming no fading is not always reasonable. This assumption is made because we wish to find the OP of MAC protocols under optimal conditions, as in [4], [5]. In [19], we add fading to our model, and derive the OP of the various protocols. Fading is shown to deteriorate the average performance of the network.

The assumption of fixed packet length $T$ is reasonable, because in most applications, data to be transmitted is packetized before transmission, and each packet is then of the same constant length. The fixed distance, $R$, between all TXRX pairs (which is also assumed in [2], [5], [7]) is often not a natural assumption. However, we know that for low 


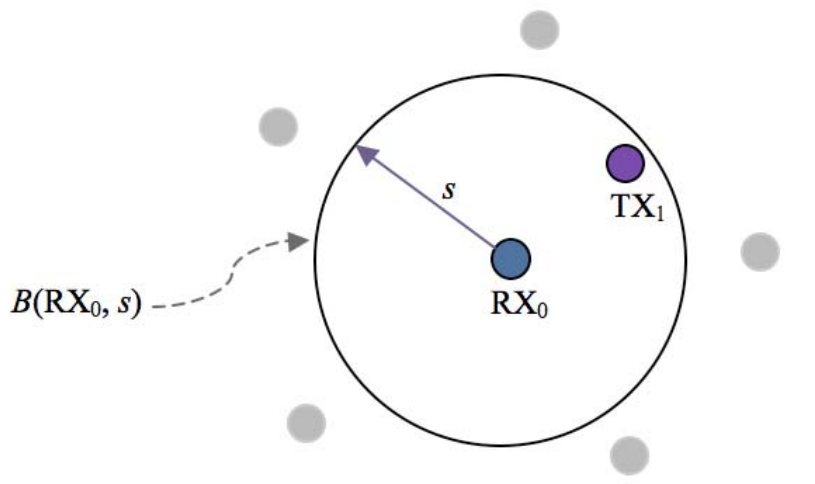

Fig. 2. When at least one interferer $\mathrm{TX}_{1}$ falls within a distance $s$ away from $\mathrm{RX}_{0}$, i.e., inside $B\left(\mathrm{RX}_{0}, s\right)$, it causes outage for $\mathrm{RX}_{0}$.

densities, the OP is a convex ${ }^{1}$ function of $R$, making our OP analysis yield a lower bound to the case when $R$ is variable. Furthermore, it has been rigorously shown in [20] that variable transmit distances do not result in fundamentally different performances. Finally, note that the whole network with a fixed $R$ could be viewed as a snapshot of a multi-hop wireless network, where $R$ is the bounded average inter-relay distance resulting from the specific routing protocol used.

\section{Outage Probability of ALOHA}

The ALOHA protocol is one of the simplest MAC algorithms for a communication network. Here, packets are transmitted to their intended RXs immediately upon their arrival, regardless of the channel conditions. In order to analyze the OP of ALOHA, we apply the concept of guard zones [4]. First, define $s$ to be the distance between a randomly selected RX on the plane, $\mathrm{RX}_{0}$, and its closest interfering TX that causes the SINR to fall just below the threshold $\beta$. By manipulation of the SINR expression, $s$ is derived to be:

$$
s=\left(\frac{R^{-\alpha}}{\beta}-\frac{\eta}{\rho}\right)^{-\frac{1}{\alpha}} .
$$

Through Eq. (4), $\beta_{\text {sens }}$ corresponds to $s_{\text {sens }}, \beta_{\text {req }}$ to $s_{r e q}$, etc. The guard zone $B\left(\mathrm{RX}_{0}, s\right)$ is a circle of radius $s$ around $\mathrm{RX}_{0}$, as illustrated in Fig. 2. One situation that would cause $\mathrm{RX}_{0}$ to go into outage is if the accumulation of powers from all the interfering nodes outside $B\left(\mathrm{RX}_{0}, s\right)$ results in the SINR at $\mathrm{RX}_{0}$ falling below the threshold $\beta$. Another situation is if at least one active $\mathrm{TX}$, other than $\mathrm{RX}_{0}$ 's own $\mathrm{TX}, \mathrm{TX}_{0}$, falls inside $B\left(\mathrm{RX}_{0}, s\right)$ at any time during the packet transmission. Considering only the latter event yields a lower bound to the OP. It has previously been shown that this lower bound is in fact fairly tight around the actual OP [5], and hence, we only focus on this bound in our analysis.

Allowing for retransmissions is equivalent to increasing the average number of packets that attempt to access the channel, when the network is in a steady state. With the assumption

\footnotetext{
${ }^{1}$ Convexity may be proven by considering the second derivative of $P_{\text {out }}$. $P_{\text {out }}$ consists of a combination of error probability expressions of the form $P_{r t}=1-e^{-k R^{2}}$, where $k$ is a function of $\lambda$ and $\beta$. Moreover, $\frac{d^{2} P_{r t}}{d R^{2}}=$ $2 k e^{-k R^{2}}\left(1-2 k R^{2}\right)$. This is $>0$, and thus $P_{r t}$ is convex, for $2 k R^{2}<1$, which is the case for low enough densities.
}

on high mobility, and by selecting the time interval from one retransmission attempt to the next (due to a backoff or retransmission) to be $t_{\text {wait }}>T$, there is no spatial and temporal correlation between the retransmission attempts. Given the probability of a packet being retransmitted in ALOHA is $P_{r t}$, the density of packets on the plane at each time instant is

$$
\lambda_{\text {aloha }}\left(P_{r t}\right)=\lambda\left(1+P_{r t}+P_{r t}^{2}+\ldots+P_{r t}^{N}\right)=\lambda \frac{1-P_{r t}^{N+1}}{1-P_{r t}} .
$$

Applying the concept of guard zones in our ad hoc network with spatial node density of $\lambda_{\text {aloha }}\left(P_{r t}\right)$, we shall now derive the OP of slotted and unslotted ALOHA in the following.

\section{A. Slotted ALOHA}

In slotted ALOHA, the time line is divided into slots of fixed duration $T$, and TXs can only start their transmissions at the beginning of the next time slot after each packet has been formed. Thus there is no partial overlap of packets, something that is intuitively expected to decrease the OP compared to unslotted algorithms. This performance improvement comes, however, at the expense of a need for synchronization. Since the system is slotted, we are only concerned with the locations of packet arrivals in each slot, which follow a homogeneous 2-D PPP with intensity $\lambda_{\text {aloha }}\left(P_{r t, s}\right)$, where $P_{r t, s}$ is the probability that a transmission attempt is unsuccessful. By properties of the PPP, the interferers of the node under observation also follow a homogeneous 2-D PPP with the same intensity. This yields the following theorem.

Theorem 1: The OP of slotted ALOHA can be lower bounded by $P_{o u t}^{l b}($ Slotted ALOHA $)=\tilde{P}_{r t, s}^{N+1}$, where $\tilde{P}_{r t, s}$ is the solution to

$$
\tilde{P}_{r t, s}=1-e^{-\lambda \frac{1-\tilde{P}_{r t, s}^{N+1}}{1-\tilde{P}_{r t, s}} \pi s_{r e q}^{2}} .
$$

Proof: Consider the active communication link $\mathrm{TX}_{0}-\mathrm{RX}_{0}$. Due to the slotting of time, only packets arriving during the last $T$ seconds start simultaneously with the one generated by $\mathrm{TX}_{0}$, and have thus the potential to result in an erroneous packet reception at $\mathrm{RX}_{0}$. Based on the concept of guard zones, we have that

$\mathbb{E}\left[\#\right.$ of interf. inside $B\left(\mathrm{RX}_{0}, s_{\text {req }}\right)$ at some $\left.t \in[-T, 0)\right]$

$$
\approx \lambda_{\text {aloha }}\left(\tilde{P}_{r t, s}\right) \pi s_{r e q}^{2}
$$

where $\lambda_{\text {aloha }}\left(\tilde{P}_{r t, s}\right)$ is given by Eq. (5). The probability of having an erroneous packet transmission in a Poisson

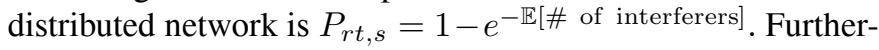
more, a packet is retransmitted the $k$-th time if it is erroneously received all $k-1$ previous attempts. Hence, a packet is counted to be in outage if it is received erroneously on the $N$-th retransmission attempt, resulting in $P_{o u t}^{l b}($ Slotted ALOHA $)=$ $\tilde{P}_{r t, s}^{N+1}$.

\section{B. Unslotted ALOHA}

In unslotted ALOHA, communication is continuous in time, i.e., packets are transmitted as soon as they are formed. Unslotted protocols are particularly of interest in systems that have no synchronization abilities. Intuitively, we expect the OP 
of unslotted ALOHA to exceed that of the slotted case, due to the partial overlap of transmissions. With the same reasoning as for slotted ALOHA, we obtain the following theorem.

Theorem 2: The OP of unslotted ALOHA can be lower bounded by $P_{o u t}^{l b}($ Unslotted ALOHA $)=\tilde{P}_{r t, u}^{N+1}$, where $\tilde{P}_{r t, u}$ is the solution to

$$
\tilde{P}_{r t, u}=1-e^{-2 \lambda \frac{1-\tilde{P}_{r t, u}^{N+1}}{1-\tilde{P}_{r t, u}} \pi s_{r e q}^{2}} .
$$

Proof: Due to the unslottedness of the system, any transmission that started less than time $T$ before the start of $\mathrm{TX}_{0}$ 's transmission and up to time $T$ later, will be interfering with the packet of $\mathrm{RX}_{0}$ and thus contribute to its OP. Since the number of packet arrivals at times $t_{0}$ and $t_{0}+T$ are independent, we have that

$$
\begin{aligned}
\tilde{P}_{r t, u} & =1-\operatorname{Pr}\left(\operatorname{No} \mathrm{TXs} \text { in } B\left(\mathrm{RX}_{0}, s_{r e q}\right) \text { during }[-T, T)\right) \\
& =1-e^{-2 \lambda_{\text {aloha }}\left(\tilde{P}_{r t, u}\right) \pi s_{r e q}^{2},}
\end{aligned}
$$

where $\tilde{P}_{r t, u}$ is the approximate probability of an erroneous packet reception in unslotted ALOHA, with $\lambda_{\text {aloha }}\left(\tilde{P}_{r t, u}\right)$ as given by Eq. (5). Given $N$ retransmissions for each packet, the OP becomes $\tilde{P}_{r t, u}^{N+1}$.

For low densities, $P_{r t, s} \approx P_{r t, u}$, allowing us to compare slotted and unslotted ALOHA. Applying the Taylor expansion, we obtain that slotted ALOHA outperforms its unslotted version by a factor of 2 . This is expected and consistent with the results obtained from the conventional model [21]. This result is related to the fact that the space-time volume in slotted ALOHA, $V_{s}=A T$, is half of that of unslotted ALOHA, $V_{u}=2 A T$, as only one slot needs to be considered in the slotted system as opposed to two in the unslotted case.

\section{Outage Probability of CSMA}

Due to the poor performance of unslotted ALOHA, a new MAC protocol, termed Carrier-Sensing Multiple Access (CSMA), was proposed by Kleinrock and Tobagi in [9] more than 30 years ago. By introducing channel sensing and the ability to back off from transmissions, the performance of wireless networks was greatly improved. Moreover, several modifications were proposed in order to overcome the inherent hidden and exposed node problems [9] of CSMA. By allowing some kind of communication between the TX and its RX, throughput improvement was achieved. In the following subsections, we extend the work of [9] to consider point-to-point wireless ad hoc networks, and we evaluate the OP performance of CSMA.

As explained in Section II, a packet is backed off if the measured or estimated (depending on whether the RX or TX are sensing) SINR is below the sensing threshold, $\beta_{\text {sens }}$, at the beginning of its transmission. Up to a maximum of $M$ times, the packet then waits a random time before the channel is sensed again and a new decision is made. Once initiated, but received in error at its RX, the packet is retransmitted. This is repeated $N$ times before the packet is dropped. These properties yield the following theorem.

Theorem 3: The total OP of CSMA may be expressed as

$$
P_{\text {out }}(\mathrm{CSMA})=P_{b}^{M}+\left(1-P_{b}^{M}\right) P_{r t 1} P_{r t}^{N} ;
$$

and the density of packets attempting to access the channel is

$\lambda_{c s m a}\left(P_{b}, P_{r t 1}, P_{r t}\right)=\lambda\left[\frac{1-P_{b}^{M}}{1-P_{b}}+\left(1-P_{b}^{M}\right) P_{r t 1} \frac{1-P_{r t}^{N}}{1-P_{r t}}\right]$,

where $P_{r t 1}$ is the probability that an activated packet is received erroneously at its first transmission attempt and must be retransmitted, and $P_{r t}$ is the probability of error in the retransmission attempts. Expressions for $P_{b}, P_{r t 1}$, and $P_{r t}$ are derived in the following subsections.

Proof: The proof of Theorem 3 is given in Appendix A.

Due to the backoff property of CSMA, and since packets tagged for retransmission do not perform new channel sensing, we multiply the first term of Eq. (10) by $\left(1-P_{b}\right)$ to find the density of active packets;

$$
\begin{aligned}
& \lambda_{\text {active }}\left(P_{b}, P_{r t 1}, P_{r t}\right) \\
&=\lambda\left(1-P_{b}^{M}+\left(1-P_{b}^{M}\right) P_{r t 1} \frac{1-P_{r t}{ }^{N}}{1-P_{r t}}\right) .
\end{aligned}
$$

In this section, we assume that the sensing threshold, $\beta_{\text {sens }}$, based on which the backoff decision is made, is constant and equal to the required SINR for correct reception of packets, $\beta_{\text {req }}$. That is, $\beta_{\text {sens }}=\beta_{\text {req }}=\beta=0 \mathrm{~dB}$, which is equivalent to $s_{\text {sens }}=s_{\text {req }}=s \approx R$. The value $0 \mathrm{~dB}$ is chosen in order for the OP to have little dependence on the path loss exponent $\alpha$.

\section{A. CSMA with Transmitter Sensing}

In the conventional CSMA protocol, which is employed in many of today's network standards, such as IEEE 802.11 and 802.16 , the $T X$ is the backoff decision maker. That is, when a new packet arrives, the TX immediately measures the aggregate interference power. If this is greater than $\left(\frac{\rho R^{-\alpha}}{\beta}-\eta\right)$, it backs off; Otherwise, it starts transmitting. Denoting this protocol by $\mathrm{CSMA}_{\mathrm{TX}}$, its OP is established by Theorem 4 .

Theorem 4: The total OP of $\mathrm{CSMA}_{\mathrm{TX}}$ is given by Eq. (9), where:

- $P_{b} \approx \tilde{P}_{b}$ is the backoff probability, and is found as the solution to

$$
\tilde{P}_{b}=1-e^{-\lambda\left(1-\tilde{P}_{b}^{M}+\left(1-\tilde{P}_{b}^{M}\right) \tilde{P}_{r t 1} \frac{1-\tilde{P}_{r t}^{N}}{1-\tilde{P}_{r t}}\right) \pi s^{2}},
$$

- $P_{r t} \approx \tilde{P}_{b}+\left(1-\tilde{P}_{b}\right) \tilde{P}_{\text {during }}^{T X}$ is the probability that an activated packet is received erroneously in a retransmission attempt, with $\tilde{P}_{\text {during }}$ being the probability that the error occurs at some $t \in(0, T)$;

$\tilde{P}_{\text {during }}^{T X}=1-e^{-\int_{s-R}^{s} \lambda_{c s m a}\left[2 \pi-2 \cos ^{-1}\left(\frac{r^{2}+R^{2}-s^{2}}{2 R r}\right)\right] r} d r$,

with $\lambda_{c s m a}=\lambda_{c s m a}\left(P_{b}, P_{r t 1}, P_{r t}\right)$ as given by Eq. (10).

- $P_{r t 1} \approx \tilde{P}_{r x \mid \text { transmit }}+\left(1-\tilde{P}_{r x \mid \text { transmit }}\right) \tilde{P}_{\text {during }}^{T X}$ is the probability that an activated packet is received erroneously at the first transmission attempt, with $\tilde{P}_{r x \mid \text { transmit }}$ being the probability that the $\mathrm{RX}$ is in outage upon the packet arrival, given its TX decides to 
transmit;

$$
\begin{aligned}
& \tilde{P}_{r x \mid \text { transmit }} \\
& \quad=\tilde{P}_{b}\left[1-\frac{1}{\pi s^{2}}\left(2 s^{2} \cos ^{-1}\left(\frac{R}{2 s}\right)-R s \sqrt{1-\frac{R^{2}}{4 s^{2}}}\right)\right] .
\end{aligned}
$$

Proof: The proof of Theorem 4 is given in Appendix B.

Due to the inter-dependence between $P_{b}, P_{d u r i n g}$, and $\lambda_{c s m a}$, their values are found through numerical iterations. Also, the reason Eqs. (12)-(14) are approximations is that the concept of guard zones is used to derive a lower bound, while the assumption that all new interferers ignore each other and make their backoff decision based on $\mathrm{TX}_{0}$ only, increases the OP above the lower bound. This is discussed further in Appendix B.

The OP of $\mathrm{CSMA}_{\mathrm{TX}}$ is due to the hidden and exposed node problems. The hidden node problem occurs when a new interferer $\mathrm{TX}_{i}$ is located inside $B\left(\mathrm{RX}_{0}, s\right) \cap \overline{B\left(\mathrm{TX}_{0}, s\right)}$, where $\mathrm{TX}_{0}$ is hidden to $\mathrm{TX}_{i}$, during all the $N$ retransmission attempts of the packet of $\mathrm{TX}_{0}-\mathrm{RX}_{0}$. However, compared to

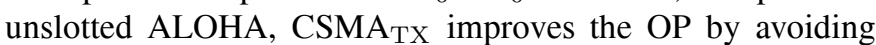
transmissions inside $B\left(\mathrm{TX}_{0}, s\right) \cap B\left(\mathrm{RX}_{0}, s\right)$. The area of this region is $A=2 s^{2} \cos ^{-1}\left(\frac{R}{2 s}\right)-R s \sqrt{1-\left(\frac{R}{2 s}\right)^{2}}$. The exposed node problem occurs when $\mathrm{TX}_{i}$ backs off in cases when its transmission would not have contributed to any outage, i.e., when $\mathrm{TX}_{i}$ is located inside $B\left(\mathrm{TX}_{0}, s\right) \cap \overline{B\left(\mathrm{RX}_{0}, s\right)}$ during all the $M$ backoffs. This adds to the OP of unslotted ALOHA. Hence, we have that

$$
\begin{aligned}
& P_{\text {out }}\left(\mathrm{CSMA}_{\mathrm{TX}}\right) \approx P_{\text {out }}(\text { Unslotted ALOHA }) \\
&-\left(1-e^{-\lambda_{\text {active }} A}\right)^{N}+\left(1-e^{-\lambda_{\text {active }}\left(\pi s^{2}-A\right)}\right)^{M},
\end{aligned}
$$

where $\lambda_{\text {active }}$ is given by Eq. (11). This approximation works best for low densities, i.e., when $\lambda_{\text {active }} \approx \lambda_{\text {aloha }}$.

To better understand the behavior of the backoff probability, let w.l.o.g. $(M, N)=(1,0) . P_{b}$ can then be expressed in terms of the Lambert function, $W_{0}(\cdot)$ [1]. Let $x=\lambda \pi s^{2}$ in Eq. (12), and apply l'Hopital's rule:

$$
\begin{aligned}
& \lim _{x \rightarrow 0} \frac{1-\frac{1}{x} W_{0}(x)}{1-e^{-x}}=\lim _{x \rightarrow 0} \frac{x-W_{0}(x)}{x\left(1-e^{-x}\right)} \\
& =\lim _{x \rightarrow 0} \frac{1-\frac{d W_{0}(x)}{d x}}{1-e^{-x}+x e^{-x}} \\
& =\lim _{x \rightarrow 0} \frac{e^{W_{0}(x)}+x-1}{\left(e^{W_{0}(x)}+x\right)\left[e^{-x}(x-1)+1\right]} \\
& =\lim _{x \rightarrow 0} \frac{1}{\left[e^{-x}(x-1)+1\right]+\frac{e^{W_{0}(x)}+x}{e^{W_{0}(x)} \frac{d W_{0}(x)}{d x}+1}\left[-e^{-x}(x-1)+e^{-x}\right]} \\
& =1 .
\end{aligned}
$$

This proves that as $\lambda \rightarrow 0, P_{b} \rightarrow P_{\text {out }}$ (Slotted ALOHA). Equivalently, for a fixed backoff probability $\bar{P}_{b}$, we have that the density of active packets in CSMA is $\lambda_{\text {active }}=$ $\frac{\lambda_{\text {aloha }}\left(\tilde{P}_{r t, s}\right)}{1-\bar{P}_{b}}$. For low $P_{b}, \lambda_{\text {active }} \approx \lambda_{\text {aloha }}$, while as $P_{b}$ increases, the density of active transmissions in CSMA may no longer be approximated by that of ALOHA. Due to the reduced number of interferers, $P_{b}$ is less than the case when all prior arrivals are activated. Hence, while Eq. (12) is an approximate measure of $P_{b}$, Eq. (6) operates as an upper bound.

\section{B. CSMA with Receiver Sensing}

With the objective of improving the performance of CSMA, we introduce a novel protocol, termed $\mathrm{CSMA}_{\mathrm{RX}}$. In this protocol, the $R X$ senses the channel and subsequently determines whether or not the packet transmission should be initiated. The communication between the TX and RX is assumed to occur over a separate 1 bit control channel, and the delay introduced by the feedback is assumed to be small and insignificant compared to the packet length. The OP of $\mathrm{CSMA}_{\mathrm{RX}}$ is given by the following theorem.

Theorem 5: The total OP of CSMARX is given by Eq. (9), where:

- $P_{b} \approx \tilde{P}_{b}$ is the backoff probability, found as the solution to Eq. (12); $P_{r t 1} \approx \tilde{P}_{d u r i n g}^{R X}$, given below;

- $P_{r t} \approx \tilde{P}_{b}+\left(1-\tilde{P}_{b}\right) \tilde{P}_{d u r i n g}^{R X}$ is the probability that an activated packet is received erroneously some time during its transmission and must thus be retransmitted;

$$
\tilde{P}_{\text {during }}^{R X}=1-e^{\left(-\int_{s-R}^{s} \int_{\nu(r)}^{2 \pi-\nu(r)} \lambda_{c s m a} P(\operatorname{active} \mid r, \phi) r d \phi d r\right)},
$$

where $\lambda_{c s m a}=\lambda_{c s m a}\left(P_{b}, P_{r t 1}, P_{r t}\right)$ is given by Eq. (10), and $P$ (active $\mid r, \phi)$ and $\nu(r)$ are:

$$
\begin{aligned}
& P(\text { active } r, \phi)=1-\frac{1}{\pi} \cos ^{-1}\left(\frac{r^{2}+2 R^{2}-s^{2}-2 R r \cos \phi}{2 R \sqrt{r^{2}+R^{2}-2 R r \cos \phi}}\right), \\
& \nu(r)=\cos ^{-1}\left(\frac{r^{2}+2 R s-s^{2}}{2 R r}\right) .
\end{aligned}
$$

Proof: The proof of Theorem 5 is given in Appendix C.

The OP of $\mathrm{CSMA}_{\mathrm{RX}}$ is due to the hidden node problem, which occurs when an interferer is located inside $B\left(\mathrm{RX}_{0}, s\right)$, while its $\mathrm{RX}$ is located in $\overline{B\left(\mathrm{TX}_{0}, s\right)}$. This is discussed further in Sections V-B and VI.

\section{Optimizing the SEnsing Threshold}

Our objective in this section is to optimize the sensing threshold, $\beta_{\text {sens }}$, of CSMA in its various incarnations, in order to minimize the OP. In the analysis thus far, we have used a constant sensing threshold, namely $\beta_{\text {sens }}=\beta_{\text {req }}=\beta=0$ $\mathrm{dB}$. In this section, we take into account variations in $\beta_{\text {sens }}$ (translating to $s_{\text {sens }}$ by Eq. (4)). For the sake of readability of the formulas, we denote $s_{\text {sens }}$ by $s$. Initially, we assume that $\beta_{\text {req }}$ stays constant (w.l.o.g., we assume that $\beta_{\text {req }}=0 \mathrm{~dB}$, which corresponds to $s_{r e q} \approx R$ ), while $\beta_{\text {sens }}$ varies. Next, in Section V-C, we set $\beta_{\text {sens }}=\beta_{\text {req }}$, and allow both the thresholds to vary.

We consider both CSMA $_{\mathrm{TX}}$ and $\mathrm{CSMA}_{\mathrm{RX}}$, and derive their OPs based on the following subsections.

\section{A. CSMA with Transmitter Sensing}

When $s$ varies, it results in changes in the area of $B\left(\mathrm{TX}_{0}, s\right)$. This impacts $P_{b}, P_{r t 1}$, and $P_{r t}$, as seen below.

Theorem 6: The total OP of $\mathrm{CSMA}_{\mathrm{TX}}$ for varying sensing thresholds is given by Eq. (9), where: 


$$
\begin{aligned}
& \tilde{P}_{\text {during }}^{T X}= \begin{cases}1-e^{-\lambda_{\text {csma }}}\left[\int_{0}^{R-s} 2 \pi r d r+\int_{R-s}^{s_{r e q}}\left[2 \pi-2 \cos ^{-1}\left(\frac{r^{2}+R^{2}-s^{2}}{2 R r}\right)\right] r d r\right] & \\
1-e^{-\lambda_{c s m a} \int_{s-R}^{s_{r e q}}\left[2 \pi-2 \cos ^{-1}\left(\frac{r^{2}+R^{2}-s^{2}}{2 R r}\right)\right] r d r} & ; R \leq s<R+s_{r e q} \\
0 & ; \text { otherwise }\end{cases} \\
& \tilde{P}_{r x \mid \text { transmit }}= \begin{cases}P_{r x}-P_{r x} \frac{s^{2}}{\pi s_{r e q}^{2}} \cos ^{-1}\left(\frac{R^{2}+s^{2}-s_{r e q}^{2}}{2 R s}\right)-P_{r x} \frac{1}{\pi} \cos ^{-1}\left(\frac{R^{2}+s_{r e q}^{2}-s^{2}}{2 R s_{r e q}}\right) & \\
\quad+\frac{P_{r x}}{2 \pi s_{r e q}^{2}} \sqrt{\left(s+s_{r e q}-R\right)\left(s-s_{r e q}+R\right)\left(-s+s_{r e q}+R\right)\left(s+s_{r e q}+R\right)} & ; s<R+s_{r e q} \\
0 & ; \text { otherwise }\end{cases} \\
& \tilde{P}_{\text {during }}^{R X}= \\
& \begin{cases}1-e^{-\lambda_{c s m a}\left[\int_{0}^{s} \int_{0}^{2 \pi} P(\operatorname{active} \mid r, \phi) r d \phi d r+2 \int_{s}^{s} s_{r e q} \int_{\nu(r)}^{\zeta(r)} P(\operatorname{active} \mid r, \phi) r d \phi d r+\int_{s}^{s} r e q[2 \pi-2(\zeta(r)-\nu(r))] r d r\right]} & ; 0<s<R \\
1-e^{-\lambda_{c s m a}\left[\int_{0}^{2 R-s} \int_{0}^{2 \pi} P(\operatorname{active} \mid r, \phi) r d \phi d r+\int_{2 R-s}^{s_{r e q}} \int_{\nu(r)}^{2 \pi-\nu(r)} P(\operatorname{active} \mid r, \phi) r d \phi d r\right]} & ; R \leq s<2 R \\
1-e^{-\lambda_{c s m a} \int_{s-2 R}^{s_{r e q}} \int_{\nu(r)}^{2 \pi-\nu(r)} P(\operatorname{active} \mid r, \phi) r d \phi d r} & ; 2 R \leq s<2 R+s_{r e q} \\
0 & ; \text { otherwise }\end{cases}
\end{aligned}
$$

- $P_{b} \approx \tilde{P}_{b}$ is given by Eq. (12); and $\tilde{P}_{r x}=1-$ $e^{-\pi \lambda_{\text {active }} s_{r e q}^{2}}$ is the approximate probability that the RX is in outage upon arrival in each retransmission attempt with $\lambda_{\text {active }}$ given by Eq. (11).

- $P_{r t} \approx \tilde{P}_{r x}+\left(1-\tilde{P}_{r x}\right) \tilde{P}_{d u r i n g}^{T X}$ is the probability that an activated packet is received erroneously in a retransmission attempt, with $\tilde{P}_{\text {during }}$ being the probability that the error occurs at some $t \in(0, T)$. This is given by Eq. (18).

- $P_{r t 1} \approx \tilde{P}_{r x \mid \text { transmit }}+\left(1-\tilde{P}_{r x \mid \text { transmit }}\right) \tilde{P}_{\text {during }}^{T X}$ is probability that the first transmission is erroneous, with $\tilde{P}_{r x \mid \text { transmit }}$ being the probability that the RX is in outage at the start of the packet. This is given by Eq. (19).

Proof: The proof of Theorem 6 is given in Appendix D.

Optimizing the sensing threshold in $\mathrm{CSMA}_{\mathrm{TX}}$ yields an optimal tradeoff between the hidden and exposed node problems, as also mentioned in Section IV-A. An increase in one problem (by changing $s$ ) leads to a decrease in the other, and vice versa. This is discussed further in Section VI.

\section{B. CSMA with Receiver Sensing}

In this section, we wish to improve the performance of $\mathrm{CSMA}_{\mathrm{RX}}$ by optimizing the sensing threshold. Following a similar analysis as in Section IV-B, we establish the following theorem.

Theorem 7: The total OP of $\mathrm{CSMA}_{\mathrm{RX}}$ for varying sensing thresholds is given by Eq. (9), where:

- $P_{b} \approx \tilde{P}_{b}$ is given by Eq. (12); $\tilde{P}_{r x}$ is given in Theorem 6; and
- $P_{r t} \approx \tilde{P}_{r x}+\left(1-\tilde{P}_{r x}\right) \tilde{P}_{d u r i n g}^{T X}$ is the probability that an activated packet is received erroneously in a retransmission attempt, with $\tilde{P}_{\text {during }}$ being the probability that the error occurs at some $t \in(0, T)$. This is given by Eq. (20), where $P($ active $\mid r, \phi)$ and $\nu(r)$ are given by Eq. (17), and $\zeta(r)=\cos ^{-1}\left(\frac{r^{2}-2 R s-s^{2}}{2 R r}\right)$.

- $P_{r t 1} \approx \tilde{P}_{r x \mid \text { transmit }}+\left(1-\tilde{P}_{r x \mid \text { transmit }}\right) \tilde{P}_{\text {during }}^{R X}$ is the probability that the first transmission attempt is erroneous, with $\tilde{P}_{r x \mid \text { transmit }}$ being the probability that the $\mathrm{RX}$ is in outage at the start of the packet;

$$
\tilde{P}_{r x \mid \text { transmit }}= \begin{cases}P_{r x}\left[1-\frac{s^{2}}{s_{r e q}^{2}}\right] & ; s<s_{r e q} \\ 0 & ; \text { otherwise }\end{cases}
$$

Proof: The proof of Theorem 7 is given in Appendix E.

Our simulation results indicate that the optimal sensing threshold in $\mathrm{CSMA}_{\mathrm{RX}}$ is $\beta_{\text {sens }}=\beta_{\text {req }}$ (equivalent to $\left.s_{\text {sens }}=s_{\text {req }}\right)$. To understand this, consider w.l.o.g. the case of $(M, N)=(1,0)$, simplifying Eq. (9) to

$$
\begin{aligned}
\tilde{P}_{\text {total }} & =\tilde{P}_{\text {out }}\left(\mathrm{CSMA}_{\mathrm{RX}}\right) \\
& = \begin{cases}\tilde{P}_{r x}+\left(1-\tilde{P}_{r x}\right) \tilde{P}_{\text {during }}^{R X} & ; s<s_{\text {req }} \\
\tilde{P}_{b}+\left(1-\tilde{P}_{b}\right) \tilde{P}_{\text {during }}^{R X} & ; \text { otherwise }\end{cases}
\end{aligned}
$$

Based on this, we now evaluate the rate of change of the different sources of outage, namely $\tilde{P}_{b}$ and $\tilde{P}_{d u r i n g}^{R X}$.

- When $s<s_{r e q}$ :

$$
\frac{d \tilde{P}_{\text {total }}}{d s}=\left[1-\tilde{P}_{r x}\right] \frac{d \tilde{P}_{d u r i n g}^{R X}}{d s} .
$$

Since $\tilde{P}_{r x}$ is only a function of $s_{r e q}$, its derivative 


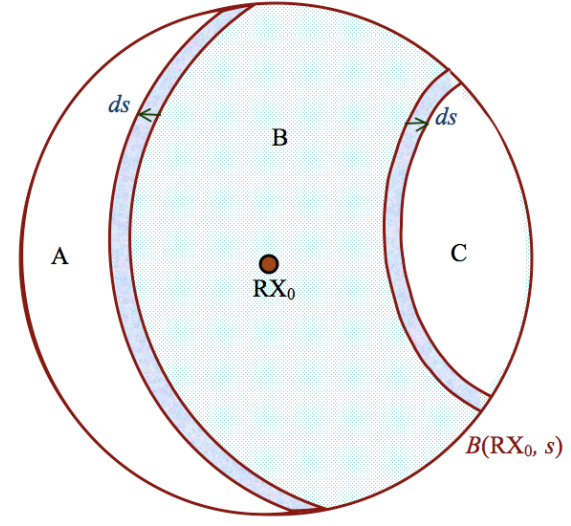

Fig. 3. The setup used in Section V-B to illustrate the rate of increase in $P_{b}$ and decrease in $P_{\text {during }}$ as $\beta$ increases.

with respect to $s$ is $0 . \tilde{P}_{d u r i n g}^{R X}$ on the other hand is a monotonically decreasing function of $s$, which can be observed by considering Fig. 3. As $s$ increases with $d s$, the areas $\mathrm{A}$ and $\mathrm{C}$ shrink. As the decrease in these areas has a greater impact on $\tilde{P}_{d u r i n g}^{R X}$ than the increase in area $\mathrm{B}$ (because in $\mathrm{A}$ and $\mathrm{C}, P($ active $\mid r, \phi)=1$ ), we get a decrease in $\tilde{P}_{\text {during }}^{R X}$. Intuitively, this means that an increase in $s$ results in more protection for an arriving TX-RX pair, resulting in a higher rate of backoff. Consequently, due to the reduced number of interferers, there is a smaller probability that a packet transmission goes into outage once it has been activated. Hence, $\frac{d \tilde{P}_{\text {total }}}{d s}<0$ for $s<s_{\text {req }}$.

- When $s \geq s_{r e q}$ :

$$
\frac{d \tilde{P}_{\text {total }}}{d s}=\left[1-\tilde{P}_{d u r i n g}^{R X}\right] \frac{d \tilde{P}_{b}}{d s}+\left[1-\tilde{P}_{b}\right] \frac{d \tilde{P}_{d u r i n g}^{R X}}{d s} .
$$

When $s$ increases by $d s, B\left(\mathrm{RX}_{0}, s\right)$ grows and so does $\tilde{P}_{b}$. The rate of this increase is:

$$
\frac{d \tilde{P}_{b}}{d s} \approx \frac{\pi(s+d s)^{2}-\pi s^{2}}{\pi s^{2}}=\frac{d s^{2}+2 s d s}{s^{2}} .
$$

$\tilde{P}_{\text {during }}^{R X}$, on the other hand, decreases with $s$. This change may be approximated by the decrease in the area around $\mathrm{RX}_{0}$ within which the occurrence of an interferer would cause outage, given by:

$$
\begin{aligned}
\frac{d \tilde{P}_{d u r i n g}^{R X}}{d s} & \approx \frac{\left(\pi s_{r e q}^{2}-\frac{\pi(s+d s-R)^{2}}{3}\right)-\left(\pi s_{r e q}^{2}-\frac{\pi(s-R)^{2}}{3}\right)}{\pi s_{r e q}^{2}} \\
& =-\frac{2(s-R) d s+d s^{2}}{3 s_{r e q}^{2}} .
\end{aligned}
$$

To obtain the sign of $\frac{d \tilde{P}_{\text {total }}}{d s}$, we make some approximations. Since $d s \ll 1$, we set $(d s)^{2} \approx 0$. Also, since $\beta_{\text {req }}=1$, and the noise is small, we have $s_{r e q} \approx R$. The largest rate of decrease of Eq. (26) is when $s=s_{r e q}+R$. This yields $\left|\frac{d \tilde{P}_{d u r i n g}^{R X X}}{d s}\right| \approx \frac{1}{3 s_{r e q}}<\frac{1}{2 s_{r e q}} \approx\left|\frac{d \tilde{P}_{b}}{d s}\right|$.

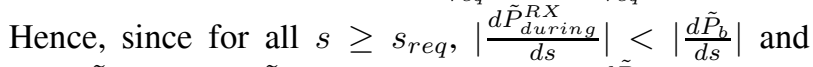
$\left(1-\tilde{P}_{b}\right) \leq\left(1-\tilde{P}_{\text {during }}^{R X}\right)$, we have that $\frac{d \tilde{P}_{\text {total }}}{d s}>0$ for $s \geq s_{\text {req }}$.

Thus, we conclude that the OP of CSMA $\mathrm{Rx}_{\text {in minimized }}$ for $\beta_{\text {sens }}^{o p t}=\beta_{\text {req }}$ (i.e., $s_{\text {sens }}^{o p t}=s_{\text {req }}$ ). Note that the reduction in the OP by using $\beta_{\text {sens }}^{\text {opt }}$ is more evident at higher densities, as will be discussed in Section VI.

\section{Dependence of OP on the Required SINR Threshold}

In this section, we assume that both the sensing threshold and the required SINR threshold are varying, while at the same time remaining equal, i.e., $\beta_{\text {sens }}=\beta_{\text {req }}=\beta$ (equivalently $s_{\text {sens }}=s_{r e q}=s$ ). The total OP of CSMA by replacing $s_{r e q}$ by $s$ in Theorem 6, with the following differences:

- The approximate probability that the RX is in outage at the start of its first transmission attempt, is:

$$
\begin{aligned}
& \tilde{P}_{r x \mid \text { transmit }}= \\
& \begin{cases}P_{r x} & ; s<\frac{R}{2} \\
P_{r x}\left[1-\frac{2}{\pi} \cos ^{-1}\left(\frac{R}{2 s}\right)+\frac{R}{\pi s} \sqrt{1-\left(\frac{R}{2 s}\right)^{2}}\right] & ; \text { otherwise }\end{cases}
\end{aligned}
$$

- The approximate probability that a packet goes into outage at some $t \in(0, T)$ is now given by Eq. (28).

The OP of CSMA $\mathrm{RX}$ is found by setting $s_{r e q}=s_{\text {sens }}=s$ in Theorem 7, with the difference that the third line in Eq. (20) is now valid for all $s \geq 2 R$.

\section{NUMERICAL RESULTS}

For the simulations, we generate, as described in Section II, the 3-D PPP of packets over an area of $A=1000 \mathrm{~m}^{2}$, and set w.l.o.g. $R$ and $\rho$ to be 1 , and the path loss exponent $\alpha=4$. The derived formulas for slotted and unslotted ALOHA are plotted in Fig. 4 for $(M, N)=(1,0),{ }^{2}$ and seen to follow the simulation results tightly for all densities. The curves confirm Theorems 1 and 2, and that the slotted system outperforms the unslotted one by approximately a factor of 2 . Moreover, we observe that the OP of ALOHA increases linearly with the number of active interferers on the plane (which is equal to the number of packet arrivals), until it reaches a saturation point where $\mathrm{OP} \approx 1$. For the sake of the discussions in Section IV-A, the backoff probability of CSMA, $P_{b}$, is also plotted in Fig. 4. For low densities, $P_{b}$ is approximately equal to the OP of slotted ALOHA. For higher densities, due to fewer active interferers in CSMA, $P_{b}<P_{\text {out }}$ (Slotted ALOHA).

Fig. 5 shows the OP performance of $\mathrm{CSMA}_{\mathrm{TX}}$ and $\mathrm{CSMA}_{\mathrm{RX}}$ for $(M, N)=(1,0)$ and $(M, N)=(2,1)$. The analytical expressions are confirmed as they are seen to follow the simulations tightly for $(M, N)=(1,0)$. However, for $(M, N)=(2,1)$, we observe a greater discrepancy. This is due to the fact that the guard zone approximation is used multiple times when $M>1$ and $N>0$. Clearly, the OP performance of CSMA is considerably reduced by increasing $M$ and $N$. In order to compare the different protocols, in Fig. 6 , the ratio of the OP of $\mathrm{CSMA}_{\mathrm{TX}}$ and $\mathrm{CSMA}_{\mathrm{RX}}$ over that

${ }^{2}$ In [22], the exact OP of slotted ALOHA for $\alpha=4$ is derived to be: $P_{\text {out }}($ Slotted ALOHA $)=1-\operatorname{erfc}\left(\sqrt{\pi \beta} \lambda \pi R^{2} / 2\right)$. Extending this to unslotted ALOHA yields: $P_{\text {out }}($ Unslotted ALOHA $)=1-$ $\operatorname{erfc}\left(\sqrt{\pi \beta} \lambda \pi R^{2} / 2\right)^{2}$. These expressions are also plotted in Fig. 4. 


$$
\tilde{P}_{\text {during }}^{T X}= \begin{cases}1-e^{-\lambda_{\text {csma }} \pi s^{2}} & ; s<\frac{R}{2} \\ 1-e^{-\lambda_{\text {csma }}}\left[\int_{0}^{R-s} 2 \pi r d r+\int_{R-s}^{s}\left[2 \pi-2 \cos ^{-1}\left(\frac{r^{2}+R^{2}-s^{2}}{2 R r}\right)\right] r d r\right] & ; \frac{R}{2}<s<R \\ 1-e^{-\lambda_{\text {csma }} \int_{s-R}^{s}\left[2 \pi-2 \cos ^{-1}\left(\frac{r^{2}+R^{2}-s^{2}}{2 R r}\right)\right] r d r} & ; \text { otherwise }\end{cases}
$$

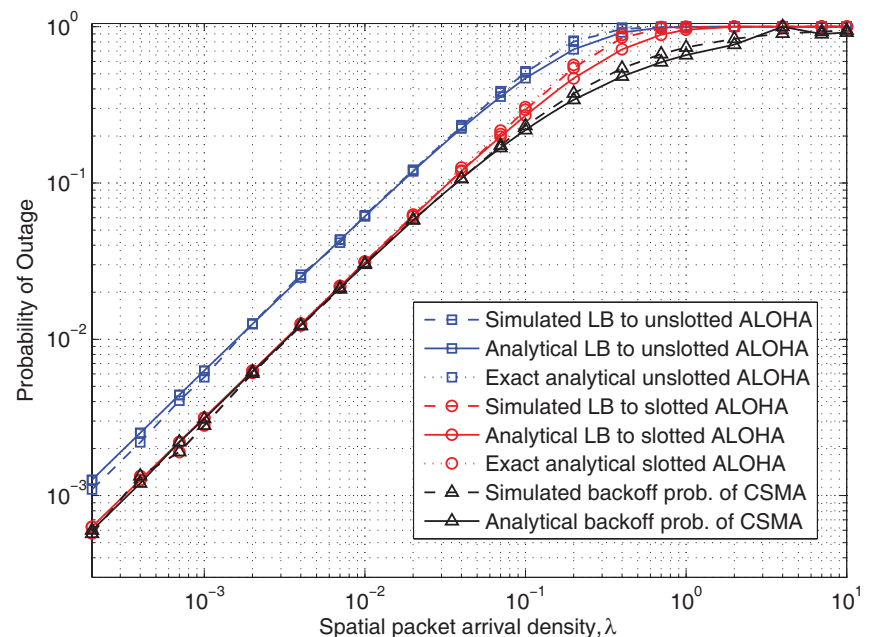

Fig. 4. OP of slotted and unslotted ALOHA along with the backoff probability of CSMA, as a function of spatial packet arrival density, for $(M, N)=(1,0)$. The curves confirm our analytical results.

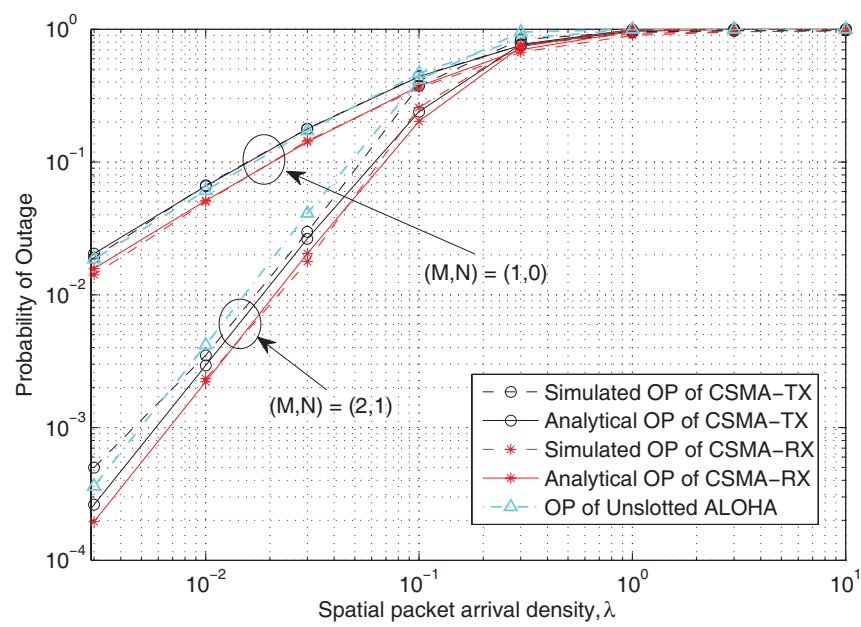

Fig. 5. OP of CSMA as a function of spatial packet arrivals density for $(M, N)=(1,0)$ and $(M, N)=(2,1)$. The curves confirm our analytical results, and show the significant improvement provided by increasing the allowable number of backoffs and retransmissions.

of slotted ALOHA for $\beta_{\text {sens }}=\beta_{\text {req }}=0 \mathrm{~dB}$ is plotted for both $(M, N)=(1,0)$ and $(M, N)=(2,1)$. Interestingly, for lower densities, $\mathrm{CSMA}_{\mathrm{TX}}$ yields about $10 \%$ more OP than unslotted ALOHA. This is due to the exposed node problem, i.e., the TX backs off in cases when its transmission would not have contributed to any outage. The performance of slotted ALOHA is almost two orders of magnitude higher than that of $\mathrm{CSMA}_{\mathrm{TX}}$, as was also concluded in [6]. As the density

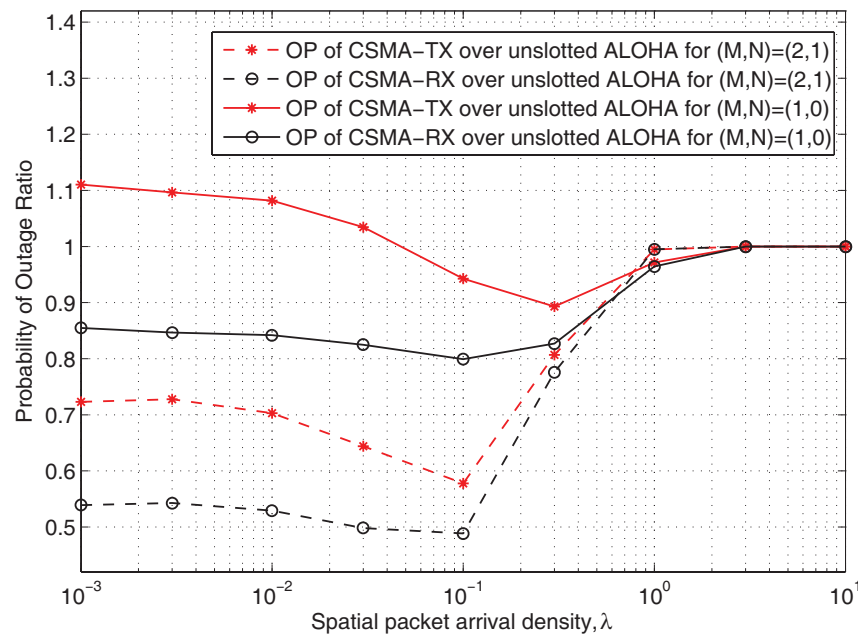

Fig. 6. Ratio of the OP of CSMA ALOHA both for when $(M, N)=(1,0)$ and $(M, N)=(2,1)$, as a function of density for a constant SINR threshold of $\beta_{\text {sens }}=\beta_{\text {req }}=0 \mathrm{~dB}$.

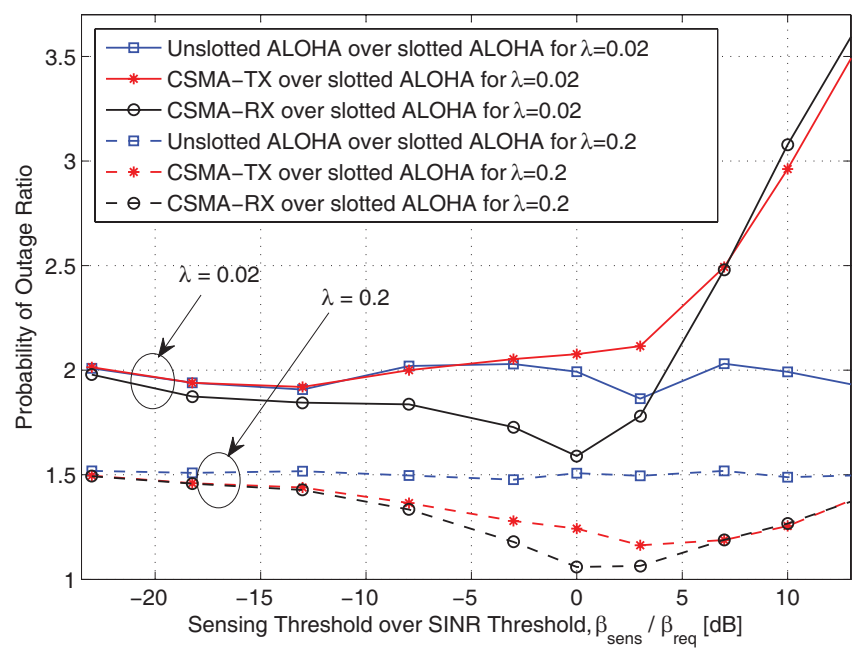

Fig. 7. Ratio of the OP of unslotted ALOHA and CSMA over that of slotted ALOHA for $(M, N)=(1,0)$, as a function of the normalized sensing threshold $\beta_{\text {sens }} / \beta_{\text {req }}$ for both a fixed low density of $\lambda=0.02$ and a fixed high density of $\lambda=0.2$.

increases, so does $P_{b}$, thus decreasing the level of interference in the channel and making CSMA more advantageous. The introduction of the feedback channel in CSMA $_{R X}$ emphasizes the advantage of the backoff property, providing a performance gain of $25 \%$ compared to CSMA $\mathrm{TX}_{\mathrm{TX}}$ when $(M, N)=(1,0)$ and $20 \%$ when $(M, N)=(2,1)$.

In Fig. 7, the ratio of the OP of CSMA over that of 


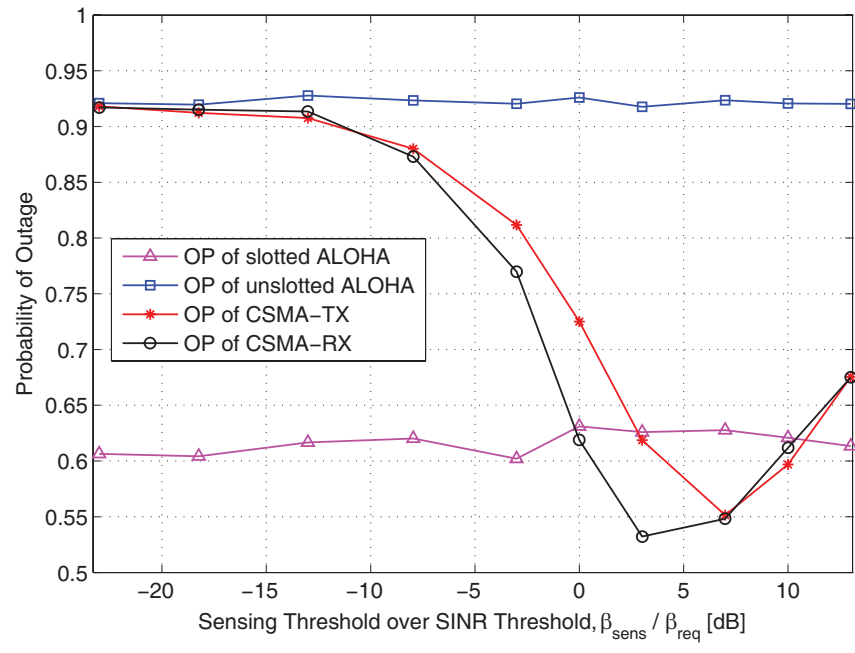

Fig. 8. OP of unslotted ALOHA and CSMA for $(M, N)=(2,1)$, as a function of the normalized sensing threshold $\beta_{\text {sens }} / \beta_{\text {req }}$ for a fixed high density of $\lambda=0.2$. Similar behavior is seen for low densities.

slotted ALOHA with $(M, N)=(1,0)$ is plotted as a function of $\beta_{\text {sens }} / \beta_{\text {req }}$, for both a low density of $\lambda=0.02$ and a high density of $\lambda=0.2$. For low densities, we observe that the OP of CSMA $\mathrm{TX}_{\mathrm{TX}}$ increases monotonically with $\beta_{\text {sens }}$, meaning that the $\mathrm{OP}$ is in fact minimized when no sensing is applied at all. This is due to the fact that for low values of $\beta_{\text {sens }}, B\left(\mathrm{TX}_{0}, s_{\text {sens }}\right)$ is too small, and consequently TXsensing provides minimal protection for its $\mathrm{RX}$, resulting in an approximately constant $\mathrm{OP}$ for all $\beta_{\text {sens }} \leq \beta_{\text {req }}$. For higher values of $\beta_{\text {sens }}, P_{b}$ becomes the dominant source of outage, and as $P_{b}$ increases at a higher rate than the decrease in $P_{\text {during }}$, the total OP increases monotonically. For $\mathrm{CSMA}_{\mathrm{RX}}$, however, a slight improvement in the OP is observed when $\beta_{\text {sens }}^{\text {opt }}=\beta_{\text {req }}$. For higher densities, the benefit of optimizing the sensing threshold becomes more significant for both protocols. The OP is minimized for $\beta_{\text {sens }} \approx \beta_{\text {req }}$ (as was also derived in Section V-B), providing up to $28 \%$ improvement for $\mathrm{CSMA}_{\mathrm{RX}}$.

Fig. 8 shows the OP performance of ALOHA and CSMA with $(M, N)=(2,1)$ for a high density of $\lambda=0.2$. The advantage of the sensing threshold optimization is more apparent when $M>1$. By using $\beta_{\text {sens }}^{o p t}$, the OP of CSMA $\mathrm{TX}_{\mathrm{TX}}$ and $\mathrm{CSMA}_{\mathrm{RX}}$ can be reduced by up to $40 \%$ and $42 \%$, respectively. Note that the minimum OP occurs at a slightly higher sensing threshold than $\beta_{r e q}$. This is because the probability that outage occurs due to the aggregate interference power from TXs outside $B\left(\mathrm{RX}_{0}, s_{r e q}\right)$ increases with $\lambda$, and having a higher $\beta_{\text {sens }}$ provides greater protection against this event.

Fig. 9 shows the ratio of the OP of unslotted ALOHA and CSMA over that of slotted ALOHA as a function of $\beta_{\text {sens }}=\beta_{\text {req }}=\beta$, for $(M, N)=(2,1)$ and a high density of $\lambda=0.2$. For low values of $\beta, \mathrm{CSMA}_{\mathrm{Rx}}$ yields up to $10 \%$ lower OP compared to unslotted ALOHA, while CSMA $\mathrm{TX}_{\mathrm{TX}}$ yields $32 \%$ higher OP. However, as $\beta$ increases (i.e., for $\beta>-6 \mathrm{~dB}$ ), making both the sensing zone and the communication zone grow, the OP of CSMA $\mathrm{TX}_{\mathrm{TX}}$ decreases below that of unslotted ALOHA. This is because the ratio of the area within which the arrival of an interferer causes outage in

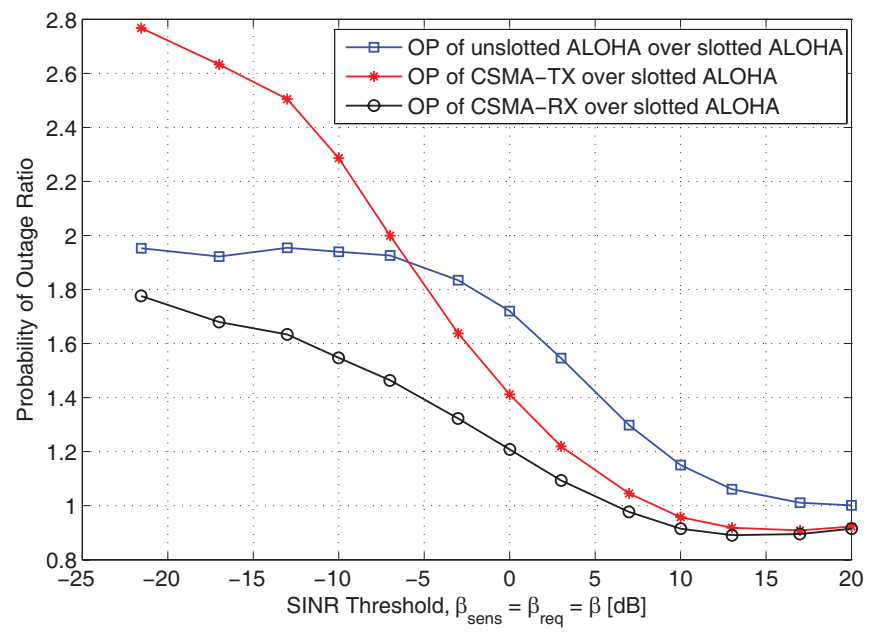

Fig. 9. Ratio of the OP of unslotted ALOHA and CSMA over that of slotted ALOHA for $(M, N)=(1,0)$, as a function of the SINR threshold $\beta_{\text {sens }}=\beta_{\text {req }}=\beta$ for a fixed high density of $\lambda=0.2$.

CSMA $_{\mathrm{TX}}$ (i.e., $\left.B\left(\mathrm{RX}_{0}, s\right) \cap \overline{B\left(\mathrm{TX}_{0}, s\right)}\right)$ over that in ALOHA (i.e., $\left.B\left(\mathrm{RX}_{0}, s\right)\right)$ decreases with $\beta$. More interestingly, for even higher values of $\beta$ (i.e., for $\beta>8 \mathrm{~dB}$ ), both CSMA protocols actually perform better than slotted ALOHA. This is because for large $s, B\left(\mathrm{TX}_{0}, s\right)$ and $B\left(\mathrm{RX}_{0}, s\right)$ overlap almost completely, such that the only source of outage in CSMA is if an interferer is placed inside $B\left(\mathrm{RX}_{0}, s\right)$ during $(0, T)$, as is the case in slotted ALOHA. Moreover, due to the backoff property of CSMA, the density of interferers is lower than that of ALOHA, making CSMA yield a lower OP. Similar behavior is observed for lower densities and other $(M, N)$-values.

\section{Conclusion and Future Research}

In this paper, we have considered the performance of the ALOHA and CSMA MAC protocols in terms of outage probability (OP). Our ad hoc network model represents a communication system in which TX-RX pairs are randomly placed on a 2-D plane, and packets arrive continuously in time based on a 1-D PPP. Within our SINR-based model, we derive expressions for the OP of slotted and unslotted ALOHA, CSMA with TX sensing $\left(\mathrm{CSMA}_{\mathrm{TX}}\right)$ and CSMA with RX sensing $\left(\mathrm{CSMA}_{\mathrm{RX}}\right)$. Our derived analytical expressions are consistent with the simulations, and an intuitive understanding is established on the benefits of CSMA over ALOHA.

An interesting result is that when no backoffs or retransmissions are allowed, CSMA $\mathrm{TX}_{\mathrm{TX}}$ actually performs worse than unslotted ALOHA for low densities due to the exposed node problem. By allowing the $R X$ to sense the channel in CSMA $\mathrm{AX}_{\mathrm{R}}$ and inform its TX over a control channel whether or not to initiate its transmission, the performance of the conventional CSMA is significantly improved.

Moreover, we optimize CSMA's sensing threshold, $\beta_{\text {sens }}$. We observe that in particular at higher densities, significant performance gain can be obtained by optimizing the sensing threshold, which is derived to be $\beta_{\text {sens }}^{\text {opt }}=\beta_{\text {req }}$. The OP of $\mathrm{CSMA}_{\mathrm{TX}}$ and $\mathrm{CSMA}_{\mathrm{RX}}$ can then be reduced by up to $40 \%$ and $42 \%$ (for $(M, N)=(2,1)$ ), respectively. This optimized sensing threshold is slightly greater than $\beta_{r e q}$ at high densities, 
due to the extra protection it provides against the aggregate interference from the TXs outside $B\left(\mathrm{RX}_{0}, s_{r e q}\right)$.

In other related works, we have investigated the impact of fading on the OP [19], and moreover optimized the OP of ALOHA and CSMA by allowing for bandwidth partitioning [23]. Other possible extensions are to apply adaptive rate and power control to improve the performance of CSMA in wireless ad hoc networks.

\section{APPENDIX}

\section{A. Proof of Theorem 3}

Denote the received SINR of the RX under observation, $\mathrm{RX}_{0}$, by $\mathrm{SINR}_{0}$. The packet transmission of $\mathrm{TX}_{0}-\mathrm{RX}_{0}$ is counted to be in outage if one or both of the following events occur:

a) The packet is backed off (i.e., $\operatorname{SINR}_{0}<\beta$ upon packet arrival) $M$ times and thus dropped.

b) Once the packet transmission is initiated, one or both of the following subevents occur $N+1$ times:

$\left.b_{1}\right) \operatorname{SINR}_{0}<\beta$ at the start of the packet, i.e., $t=0$.

$\left.b_{2}\right) \operatorname{SINR}_{0}<\beta$ at some $t \in(0, T)$.

where events $(a)$ and $(b)$ are independent except at the first transmission attempt. This yields:

$$
\begin{array}{r}
P_{\text {out }}(\mathrm{CSMA})=\operatorname{Pr}(a)+(1-\operatorname{Pr}(a)) \operatorname{Pr}\left(b_{1} \cup b_{2} \mid \bar{a}\right)^{N+1} \text { (29) } \\
=\operatorname{Pr}(a)-(1-\operatorname{Pr}(a)) \operatorname{Pr}\left(b_{1} \cup b_{2}\right)^{N} \operatorname{Pr}\left(b_{1} \cup b_{2} \mid \bar{a}\right),
\end{array}
$$

where the probability of events $(a)$ and $(b)$ are derived in the following appendices. Eq. (10) is derived as

$\lambda_{\text {csma }}\left(P_{b}, P_{r t 1}, P_{r t}\right)=$
$\begin{cases}\lambda \sum_{m=0}^{M-1} P_{b}^{m} & ; \text { for } N=0 \\ \lambda\left[\sum_{m=0}^{M-1} P_{b}^{m}+\left(1-P_{b}^{M}\right) P_{r t 1} \sum_{n=0}^{N-1} P_{r t}^{n}\right] & ; \text { for } N \geq 1\end{cases}$

\section{B. Proof of Theorem 4}

Based on Eq. (29), the probability that event ( $a$ ) occurs is $\operatorname{Pr}(a) \approx P_{b}^{M}$, where $P_{b}$ can be lower bounded by considering packet arrivals inside $B\left(\mathrm{TX}_{0}, s\right)$ during $[-T, 0)$. We assume that the number of active interferers on the plane follows a PPP (which is proven by simulation results to be reasonable) with density $\lambda_{\text {active }}$, as given by Eq. (11). Applying the OP expression for PPPs, $1-e^{-\mathbb{E}[\# \text { of active interferers] }}$, we reach Eq. (12).

Event $b_{1}$ is concerned with packet arrivals during $[-T, 0)$, resulting in $\operatorname{Pr}\left(b_{1}\right) \approx P_{b}$. For the first transmission attempt, $\operatorname{Pr}\left(b_{1} \mid a\right)$ is found geometrically as the ratio of the area of $B_{2}=B\left(\mathrm{RX}_{0}, s\right) \cap \overline{B\left(\mathrm{TX}_{0}, s\right)}$ over the area of $B\left(\mathrm{RX}_{0}, s\right)$, derived to be Eq. (14). For all retransmissions, $\operatorname{Pr}\left(b_{1} \mid a\right)=$ $\operatorname{Pr}\left(b_{1}\right)=P_{\text {during }}^{T X} \cdot \operatorname{Pr}\left(b_{2}\right)$ is lower bounded by the probability that one or more interfering TXs are located and activated inside $B\left(\mathrm{RX}_{0}, s\right)$ at some $t \in(0, T)$. We assume that all interferers (following a PPP with density $\lambda_{c s m a}$ ) base their backoff decision on the interference they see only from $\mathrm{TX}_{0}$. Since $\frac{\rho d^{-\alpha}}{\eta+\text { interf. from } \mathrm{TX}_{0}} \geq \frac{\rho d^{-\alpha}}{\eta+\text { interf. from } \mathrm{TX}_{0} \text { and all other TXs }}$ :

$\operatorname{Pr}\left[\geq 1\right.$ interferer in $B\left(\mathrm{RX}_{0}, s\right)$ at some $t \in(0, T) \mid$ active $]$ $\leq \operatorname{Pr}\left[\geq 1\right.$ interferer in $B\left(\mathrm{RX}_{0}, s\right)$ at some $t \in(0, T)$ if backoff decision only considers $\mathrm{TX}_{0} \mid$ active].

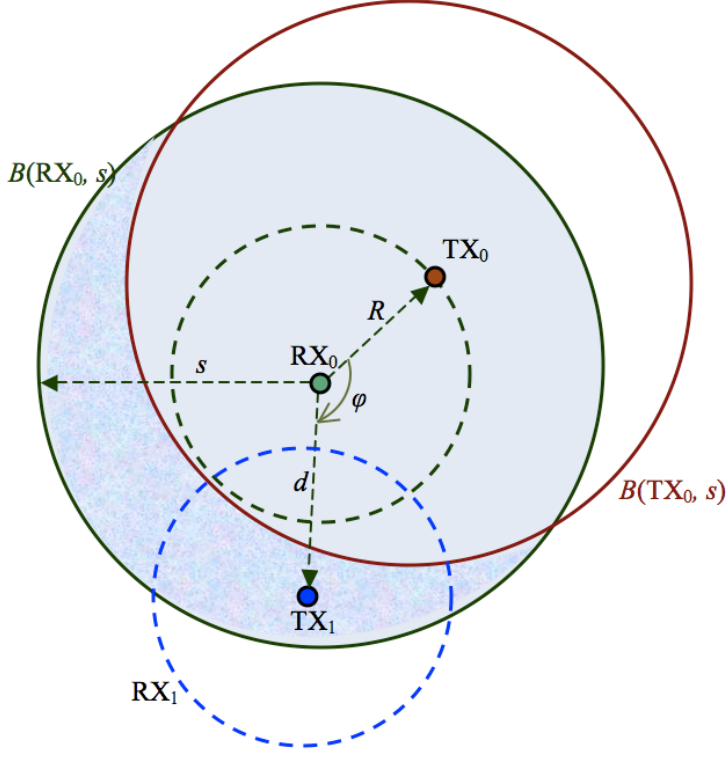

Fig. 10. The setup used to analyze $P_{\text {during }}^{T X}$ and $P_{\text {during }}^{R X}$ in Appendix B and $\mathrm{C}$, respectively. The transmission between $\mathrm{TX}_{0}$ and $\mathrm{RX}_{0}$ is assumed to be active when the new packet arrival of $\mathrm{TX}_{1}-\mathrm{RX}_{1}$ occurs.

This means that we no longer have a lower bound, but rather an approximate measure to $\operatorname{Pr}\left(b_{2}\right)$. For an interferer to be activated, it must be placed at least a distance $s$ away from $\mathrm{TX}_{0}$. Hence, $\operatorname{Pr}\left(b_{2}\right)=P_{\text {during }}^{T X}$ is derived by considering the area $B_{2}=B\left(\mathrm{RX}_{0}, s\right) \cap \overline{B\left(\mathrm{TX}_{0}, s\right)}$ (the shaded region in Fig. $10)$ :

$\mathbb{E}\left[\#\right.$ of interferers in $\left.B_{2}\right]=\int_{s-R}^{s} \int_{\gamma(r)}^{2 \pi-\gamma(r)} \lambda_{c s m a} r d \phi d r$

where $\gamma(r)$ in the integration limit is found by using the cosine-rule: $s^{2}=r^{2}+R^{2}-2 R r \cos (\gamma) \Rightarrow \gamma(r)=$ $\cos ^{-1}\left(\frac{r^{2}+R^{2}-s^{2}}{2 R r}\right)$. Solving the integral of Eq. (30) with

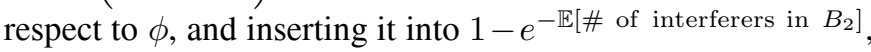
yields Eq. (13). Inserting these expressions back into Eq. (29) yields Theorem 4.

\section{Proof of Theorem 5}

As in $\mathrm{CSMA}_{\mathrm{TX}}$, we have that $\operatorname{Pr}(a) \approx P_{b}^{M}$. In order to derive $\operatorname{Pr}\left(b_{2}\right)=P_{\text {during }}^{R X}$, we apply the fact that the process that a packet starts in $B\left(\mathrm{RX}_{0}, s\right)$ in $(0, T)$ is a nonhomogeneous PPP with intensity $\mu(x, y)$.

$\mu(x, y)=\operatorname{Pr}[$ pkt arrives at $(\mathrm{x}, \mathrm{y})] \cdot \operatorname{Pr}[$ pkt activated $\mid(\mathrm{x}, \mathrm{y})]$ $=\lambda_{c s m a}^{R X} \cdot \operatorname{Pr}[\operatorname{active} \mid(\mathrm{x}, \mathrm{y})]$.

Again, we assume that all interferers base their backoff decision only on the interference from $\mathrm{TX}_{0}$, i.e., outage occurs if an interferer falls inside $B_{3}=B\left(\mathrm{RX}_{0}, s\right) \cap \overline{B\left(\mathrm{TX}_{0}, s-R\right)}$. Integrating $\mu(x, y)$ over $B_{3}$, yields:

$\mathbb{E}\left[\#\right.$ of interf. in $\left.B_{3} \operatorname{during}(0, T)\right]=\iint_{B_{3}} \mu(x, y) d x d y$

$$
=\int_{s-R}^{s} \int_{\nu(r)}^{2 \pi-\nu(r)} \lambda_{c s m a}^{R X} P(\text { active } \mid r, \phi) r d r d \phi .
$$




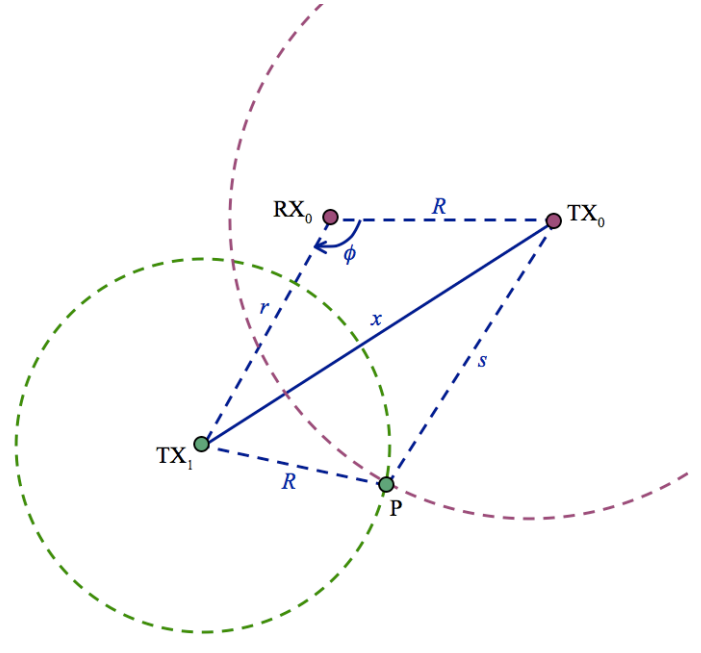

Fig. 11. The setup used in the derivation of the OP expressions for CSMA $\mathrm{TX}_{\mathrm{TX}}$ and $\mathrm{CSMA}_{\mathrm{RX}}$ in Appendix B and C, respectively.

$\nu(r)$ is found by using the cosine rule as described in Appendix B. $P($ active $\mid r, \phi)$ is the probability that $\mathrm{TX}_{i}$ initiates its transmission, and is in effect a thinning process of the rate of packet arrivals. Consider Fig. 11, and the triangle $\mathrm{TX}_{1}-\mathrm{RX}_{0}-\mathrm{TX}_{0}$. Using the cosine rule, we have: $x=\sqrt{d^{2}+R^{2}-2 R d \cos \phi}$. Next, consider the triangle P$\mathrm{TX}_{1}-\mathrm{TX}_{0}$. Again by applying the cosine rule, we derive $\theta$ to be: $\theta=\cos ^{-1}\left(\frac{x^{2}+R^{2}-s^{2}}{2 R x}\right)$. Furthermore, $\mathrm{RX}_{i}$ must be placed outside of $B\left(\mathrm{TX}_{0}, s\right)$. Thus, the probability that an interfering packet is activated is $P$ (active $\mid r, \phi)=\frac{2 \pi-2 \theta}{2 \pi}$, as given in Eq. (17). Inserting these expressions back into Eq. (31), and using the OP expression $1-e^{-\mathbb{E}\left[\# \text { of interferers in } B_{3}\right]}$, we arrive at Theorem 5.

\section{Proof of Theorem 6}

Similar to Section IV-A, once a packet transmission has been activated, we have that:

$$
\begin{gathered}
\tilde{P}_{\text {during }}^{T X} \approx \operatorname{Pr}\left[\geq 1 \text { interferer active inside } B\left(\mathrm{RX}_{0}, s_{\text {req }}\right) \cap\right. \\
\left.\overline{B\left(\mathrm{TX}_{0}, s\right)} \text { at some } t \in(0, T)\right] .
\end{gathered}
$$

This probability varies with $s$, as is reflected in the integration limits. For $R \leq s<R+s_{\text {req }}$, the derivation is as explained in Appendix B. For $s>R+s_{r e q}, B\left(\mathrm{TX}_{0}, s\right)$ covers $B\left(\mathrm{RX}_{0}, s_{r e q}\right)$, meaning that it is impossible for an interferer, $\mathrm{TX}_{i}$, to fall inside $B\left(\mathrm{RX}_{0}, s_{r e q}\right)$ and be activated. Finally, for $s<R, \mathrm{TX}_{i}$ can in addition to the area that is covered by the expression for $R \leq s<R+s_{\text {req }}$, also fall inside a circle of radius $(s-R)$ around $\mathrm{RX}_{0} . P_{r x \mid \text { transmit }}$ is the probability that at least one interferer is placed inside $B\left(\mathrm{RX}_{0}, s_{r e q}\right) \cap \overline{B\left(\mathrm{TX}_{0}, s\right)}$, given by $P_{r x} \times(1-$ area of overlap $)$. With these additional considerations to Appendix B, we arrive at Theorem 6.

\section{E. Proof of Theorem 7}

To derive $\tilde{P}_{\text {during }}^{R X}$, we consider the occurrence of an interferer, $\mathrm{TX}_{i}$, inside $B\left(\mathrm{RX}_{0}, s_{r e q}\right)$ at some $t \in(0, T)$, while $\mathrm{RX}_{i}$ is placed outside of $B\left(\mathrm{TX}_{0}, s\right)$. When $s>2 R+s_{r e q}$,
$B\left(\mathrm{TX}_{0}, s\right)$ covers $B\left(\mathrm{RX}_{0}, s_{r e q}\right)$ with a margin $R$. This means that if $\mathrm{TX}_{i}$ falls anywhere inside $B\left(\mathrm{RX}_{0}, s_{\text {req }}\right), \mathrm{RX}_{i}$ will be inside $B\left(\mathrm{TX}_{0}, s\right)$, and $\mathrm{TX}_{i}-\mathrm{RX}_{i}$ would thus back off. For the other ranges of $s$, the integration limits are adjusted as to cover the area $B_{4}(s)=B\left(\mathrm{RX}_{0}, s\right) \cap \overline{B\left(\mathrm{TX}_{0}, s-R\right)}$, in the same manner as described in Appendix $\mathrm{C}$.

Furthermore, $\tilde{P}_{r x \mid \text { transmit }}$ is derived as the probability that at least one interferer is placed inside $B_{5}=B\left(\mathrm{RX}_{0}, s_{r e q}\right) \cap$ $\overline{B\left(\mathrm{RX}_{0}, s\right)}$, and is hence zero when $s_{r e q}<s$. When $s_{r e q} \geq s$, it is equal to $\tilde{P}_{r x} \frac{B_{5}}{B\left(\mathrm{RX}_{0}, s_{r e q}\right)}$ With these additional considerations to the proof in Appendix C, we obtain Theorem 7.

\section{REFERENCES}

[1] M. Kaynia and N. Jindal, "Performance of ALOHA and CSMA in spatially distributed wireless networks," in Proc. IEEE International Conf. on Communications (ICC), pp. 1108-1112, Beijing, China, May 2008.

[2] M. Haenggi, "Outage, local throughput, and capacity of random wireless networks," IEEE Trans. Wireless Commun., vol. 8, pp. 4350-4359, Aug. 2009.

[3] N. Jindal, J. Andrews, and S. Weber, "Optimizing the SINR operating point of spatial networks," in Proc. Workshop on Info. Theory and its Applications, San Diego, CA, Jan. 2007.

[4] A. Hasan and J. G. Andrews, "The guard zone in wireless ad hoc networks," IEEE Trans. Wireless Commun., vol. 6, no. 3, pp. 897-906, Dec. 2005.

[5] S. P. Weber, X. Yang, J. G. Andrews, and G. de Veciana, "Transmission capacity of wireless ad hoc networks with outage constraints," IEEE Trans. Inf. Theory, vol. 51, no. 12, pp. 4091-4102, Dec. 2005.

[6] G. Ferrari and O. Tonguz, "MAC protocols and transport capacity in ad hoc wireless networks: Aloha versus PR-CSMA," in Proc. IEEE Military Communications Conf., Boston, USA, vol. 2, pp. 1311-1318, Oct. 2003.

[7] P. Gupta and P. R. Kumar, "The capacity of wireless networks," IEEE Trans. Inf. Theory, vol. 46, no. 2, pp. 388-404, Mar. 2000.

[8] L.-L. Xie and P. R. Kumar, "On the path-loss attenuation regime for positive cost and linear scaling of transport capacity in wireless networks," IEEE Trans. Inf. Theory, vol. 52, pp. 2313-2328, June 2006.

[9] L. Kleinrock and F. A. Tobagi, "Packet switching in radio channelspart I: carrier sense multiple-access modes and their throughput-delay characteristics," IEEE Trans. Commun., vol. 23, pp. 1400-1416, Dec. 1975.

[10] R. Vaze, "Throughput-delay-reliability tradeoff with ARQ in wireless ad hoc networks," available on http://arxiv.org/abs/1004.4432, Apr. 2010.

[11] X. Wang and K. Kar, "Throughput modelling and fairness issues in CSMA/CA based ad-hoc networks," in Proc. INFOCOM, Miami, FL, Mar. 2005.

[12] M. Garetto, T. Salonidis, and E. Knightly, "Modeling per-flow throughput and capturing starvation in CSMA multi-hop wireless networks," in Proc. INFOCOM, Barcelona, Spain, Apr. 2006.

[13] P. Mühlethaler and A. Najid, "Throughput optimization in multihop CSMA mobile ad hoc networks," in Proc. European Wireless Conf., Feb. 2004

[14] J. Zhu, X. Guo, L. Yang, and W. Conner, "Leveraging spatial reuse in 802.11 mesh networks with enhanced physical carrier sensing," in Proc. IEEE International Conf. on Communications (ICC), pp. 4004 4011, 2004.

[15] J. A. Fuemmeler, N. H. Vaidya, and V. V. Veeravalli, "Selecting transmit powers and carrier sense thresholds for CSMA protocols," University of Illinois at Urbana-Champaign Technical Report, Oct. 2004.

[16] B. J. B. Fonseca, "A distributed procedure for carrier sensing threshold Adaptation in CSMA-based mobile ad hoc networks," in Proc. Vehicular Technology Conf. (VTC), pp. 66-70, Baltimore, MD, Oct. 2007.

[17] R. K. Ganti and M. Haenggi, "Spatial and temporal correlation of the interference in Aloha ad hoc networks," IEEE Commun. Lett., vol. 13, no. 9, pp. 631-633, Sep. 2009.

[18] J. F. C. Kingman, Poisson Processes. Oxford University Press, Oxford, 1993.

[19] M. Kaynia, G. E. Øien, and N. Jindal, "Impact of fading on the performance of ALOHA and CSMA," in Proc. IEEE International Workshop on Signal Processing Advances for Wireless Communications (SPAWC), pp. 394-398, June 2009.

[20] D. Bertsekas and R. Gallager, Data Networks, chapter 4. Prentice-Hall Inc., 1987 
[21] N. Abramson, "The ALOHA system - another alternative for computer communications," in Proc. Fall Joint Computer Conf., pp. 281-296, Montvale, NJ, Nov. 1970.

[22] E. S. Sousa and J. A. Silvester, "Optimum transmission ranges in a direct-sequence spread-spectrum multihop packet radio network," IEEE J. Sel. Areas Commun., vol. 8, no. 5, pp. 762-771, June 1990.

[23] M. Kaynia, G. E. Øien, N. Jindal, and D. Gesbert, "Comparative performance evaluation of MAC protocols in ad hoc networks with bandwidth partitioning," in Proc. IEEE Inter. Symp. on Personal, Indoor and Mob. Radio Comm. (PIMRC), pp. 1-6, Sep. 2008.

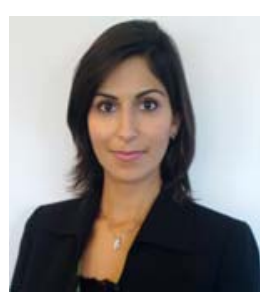

Mariam Kaynia has since August 2007 been a Ph.D. student at the Norwegian University of Science and Technology (NTNU) in Trondheim, Norway. She received her M.Sc. degree in Electrical Engineering from both NTNU and University of Minnesota in June 2007. In 2009-2010, she was a visiting researcher at Stanford University, CA, USA, and at Institut Eurecom, Sophia-Antipolis, France. She has also held shorter visiting appointments at University of Bologna and Politecnico di Torino. Her research interests are related to resource allocation in wireless ad hoc and sensor networks through MAC and PHY layer design, and specifically MAC protocols in ad hoc networks and MIMO interference channels. Miss Kaynia is a Fulbright Scholar. She was awarded the "Best IT Student of the Year" title in 2007, and she received the "Best Paper Award" at the WCSP conference in 2009. She serves in the steering committee of IEEE Norway as Treasurer and is the chair of IEEE Norway Student Branch.

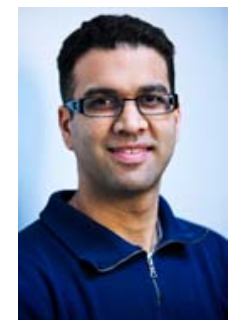

Nihar Jindal (S'99-M'04) received the B.S. degree in electrical engineering and computer science from the University of California at Berkeley in 1999 and the M.S. and Ph.D. degrees in electrical engineering from Stanford University, Stanford, CA, in 2001 and 2004, respectively. He is an Associate Professor at the Department of Electrical and Computer Engineering, University of Minnesota, Minneapolis. His industry experience includes internships at Intel Corporation, Santa Clara, CA, in 2000 and at Lucent Bell Labs, Holmdel, NJ, in 2002. His research spans the fields of information theory and wireless communication, with specific interests in multiple-antenna/multiuser channels, dynamic resource allocation, and sensor and ad hoc networks.

Dr. Jindal currently serves as an Associate Editor for the IEEE TRANSACTIONS ON COMMUNICATIONS, and was a Guest Editor for a special issue of the EURASIP Journal on Wireless Communications and Networking on the topic of multiuser communication. He was the recipient of the 2005 IEEE Communications Society and Information Theory Society Joint Paper Award, the University of Minnesota McKnight Land-Grant Professorship Award in 2007, the NSF CAREER award in 2008, and the best paper award for the IEEE JOURNAL ON SELECTED AREAS IN COMMUNICATIONS in 2009.

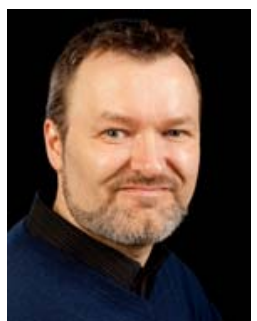

Geir E. Øien was born in Trondheim, Norway in 1965. He received the MScEE and the Ph.D. degrees, both from the Norwegian Institute of Technology (NTH) in Trondheim, Norway, respectively in 1989 and 1993. In 1996, he joined The Norwegian University of Science and Technology (NTNU) as Associate Professor, and in 2001 was promoted to Full Professor. During the academic year 20052006 he was visiting professor with Institut Eurecom in Sophia-Antipolis, France. From August 2009 to August 2013 he is on leave from his professorship in order to serve as Dean of NTNU's Faculty of Information Technology, Mathematics, and Electrical Engineering. Prof. ien's current research interests are in communication theory, information theory, and signal processing for wireless communications and sensor networks, with particular emphasis on radio resource allocation, dynamic spectrum sharing, interference management, multiple access schemes, and cross-layer design. 